\title{
Geometric Analysis of Shapes in Ion Mobility-Mass Spectrometry
}

Jean R. N. Haler ${ }^{1,2, \dagger^{*}}$, Eric Béchet ${ }^{3, \dagger}$, Johann Far ${ }^{l}$, Edwin De Pauw ${ }^{l}$

${ }^{1}$ Mass Spectrometry Laboratory, University of Liège, MolSys Research unit, Quartier Agora, Allée du Six Aout 11, B-4000 Liège, Belgium

${ }^{2}$ Department of Chemistry and Biochemistry, Florida International University, Miami, Florida 33199, USA

${ }^{3}$ Aerospace \& Mechanical Engineering Department, Computer-aided Geometric Design, University of Liège, Belgium

${ }_{\dagger}^{\dagger}$.R.N. Haler and E. Béchet equally contributed to this work

*Corresponding author email address: jean.haler@uliege.be

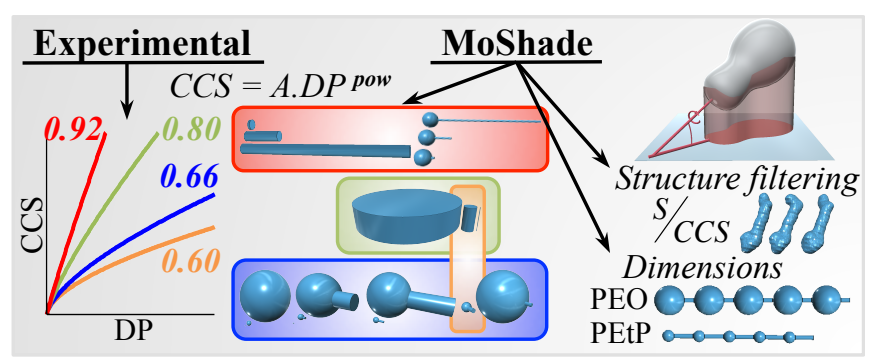

Keywords: Ion Mobility-Mass Spectrometry, Synthetic polymers, structure interpretation, Collision cross-section fitting, MoShade 


\begin{abstract}
$\underline{\text { Abstract }}$
Experimental ion mobility-mass spectrometry (IM-MS) results are often correlated to three-dimensional structures owing to theoretical chemistry calculations. The bottleneck of this approach is the need for accurate values, both experimentally and theoretically predicted. Here, we analyze experimental and theoretical collision cross-section (CCS) evolutions instead of interpreting absolute CCS values. Experimentally, the CCS trends of synthetic homopolymers are analyzed as a function of increasing degrees of polymerization (DP) for different charge states. Then, shape evolutions of modeled shape deformations yield theoretical CCS trends, calculated using new software called MoShade (projected area calculations). The shapes are modeled using computer-aided design software where we considered only geometric factors: no atoms, chemical potentials or interactions are taken into consideration to make the method orthogonal to classical methods for 3D shape assessments using time-consuming computational chemistry. We are able to correlate modeled shape evolutions to experimentally-obtained polymer CCS trends. We thus modeled the apparent volume or envelope of their ion-drift gas interactions as sampled by IM-MS. Moreover, the CCS of convex shapes could be directly related to their surface area. The relation seems to hold even for concave shapes which could be correlated to geometry-optimized structures of ions obtained by conventional computational chemistry methods. Modeling beads-on-a-string shape evolutions allows extracting precise dimension relations between two homopolymers, without modeling any chemical interactions.
\end{abstract}




\section{Introduction}

Ion Mobility-Mass Spectrometry (IM-MS) is being increasingly used for structural characterization of small molecules ${ }^{1,2}$, biomolecule folds ${ }^{3-5}$ or even synthetic polymer folds in the gas phase ${ }^{6-10}$. Nevertheless, the two-dimensional shape information yielded by IM-MS as reduced ion mobility $\left(\mathrm{K}_{0}\right)$ or Collision Cross-Section (CCS) is very often insufficient for detailed structural characterizations. Therefore, theoretical chemistry calculations are usually coupled to the IM-MS experiments. Density Functional Theory (DFT), Molecular Mechanics (MM), Molecular Dynamics (MD) or different couplings of these structure calculation methods are applied to provide detailed three-dimensional structures of the measured systems ${ }^{8-13}$. The best experiment-theory CCS value agreement $^{14}$ (often empirically fixed at $\leq 5 \%$ deviation) is then used as criterion to choose the three-dimensional structure(s) representing the ion shape sampled by IM-MS. Structural interpretations can then be undertaken.

However, there is neither a full consensus on how to calculate these three-dimensional structures (DFT, MM, MD, choice of force fields, choice of charge descriptions...), nor on how to calculate the CCS values ${ }^{15-21}$. The major issues are the unknown experimental (ion) temperatures (i.e. the ion's internal energy and the ion-drift gas interactions) in IM$\mathrm{MS}^{22-25}$ and the rapidly increasing computational cost of the calculations when the analyzed systems increase in size (number of electrons or number of atoms), which limits the affordability of detailed theoretical descriptions ${ }^{15-21}$. Furthermore, additional developments in experimental resolving power ${ }^{6,26-30}$ will increase the need for detailed theoretical descriptions of small effects such as dipole moments yielding non-negligible dipole-neutral (ion-drift gas) interactions ${ }^{11,31,32}$. 
Regarding the CCS calculations, several different algorithms have been developed ${ }^{15-21,33}$. They differ in computational $\operatorname{cost}^{34}$ as well as in the detailed descriptions of the interactions between the charged ions and the neutral drift gas particles. The CCS calculation algorithms range from calculating ion projections (shadows) to calculating detailed trajectories of the drift gas particles colliding with the ions, taking into account different levels of long-range potentials (e.g. Lennard-Jones potential), dipole-neutral interactions etc..$^{16,19,35}$.

Throughout the process of coupling experimental and theoretical results, the issue of comparing absolute CCS values arises. Both the experimental accurateness of CCS values $^{7,36,37}$ as well as the correct theoretical description of the system and its interactions are then of crucial importance. Here, we develop a new way of coupling theoretical to experimental results by avoiding absolute CCS value comparisons. This leads to an orthogonal and analytically robust shape interpretation methodology for IM-MS. For this purpose, we based our study on synthetic polymers where many data points can be generated (by increasing the polymer chain length, Degree of Polymerization, DP) without changing the essence of the interactions of the polymer-cation complexes. Experimental CCS evolutions are then interpreted through a fitting method ${ }^{38}$. Experimental fit parameters are then compared to fit parameters from CCS trends obtained from modeled structure evolutions. Modeled CCS evolutions are obtained through a new CCS calculation program named MoShade. Our MoShade calculations are purely geometric shape-based analyses with no 3D structures from theoretical chemistry intervening in the process. Shape geometries defining the fit parameters (CCS evolutions) 
can be extracted and conclusions which could also be applicable to non-polymer systems are found.

\section{Materials and Methods}

\section{Polymers and Ion Mobility-Mass Spectrometry.}

Details can be found in the Supporting Information.

\section{MoShade.}

MoShade is written in $\mathrm{C}++$ and is free software (licensed GPLv2+). MoShade is crossplatform compiled for Linux and Windows (64-bit). It is a multi-threaded, terminal-based (or command prompt) program. The shape inputs are stereolithography .stl or composite .gm files. MoShade calculates the volume, the surface area and the CCS (arbitrary units) of a shape. It also calculates the ratio of the surface area and the CCS, and the minimum and maximum CCS values from all projection angles can be printed (optional). A concavity factor is given by calculating the ratio between the sum of the reflex angles and the sum of all angles, taken at the edges of each triangle. Its value ranges from 0 (perfectly convex shape) to 1 . This concavity factor depends on the number of triangles and cannot be compared between shapes with differing numbers of triangles.

MoShade can be compiled (on Linux) with or without graphical visualization interface (using VTK or FLTK). The graphical visualization allows identifying unconnected triangles or even holes in the input structures which could possibly yield incorrect results or which increase the duration of the calculation through the loss of calculation optimizations. 
The calculations were performed using optimized integrals and using $\mathrm{n}=15$ samples, representing $\mathrm{N}=900$ projection calculations $\left(\mathrm{N}=(2 \mathrm{n})^{2}\right.$; for explanations see section below). They were sufficient for yielding invariable MoShade results; verified by calculating $\mathrm{N}=3600$ projections $(\mathrm{n}=30)$ for several structures.

MoShade (and nutil) can be downloaded at the following links:

https://cadxfem.org/svn/cadxfem/moshade/

https://cadxfem.org/svn/cadxfem/nutil/

https://github.com/JeanRNH/MoShade/releases

\section{MoShade mathematical background.}

A complete demonstration of the mathematics for (any) concave shapes can be found in the dedicated Supporting Information file. Additional developments for convex shapes are also developed.

In brief, MoShade calculates the projected area of the shape by sampling its orientation based on the angle $\theta$ and the rotation around the axis $\phi$ (Figure 1). Eq. 1 yields the average projected area $\bar{S}_{p}$ (in other words the CCS). $\bar{S}_{p}$ is the integral of the crosssection (projected area) for each orientation $(\theta, \phi)$, normalized (or weighted) by the probability to find the shape in one orientation or the other. This probability depends on the solid angle covered by an infinitesimal area on the unit sphere. This area is $2 \pi \cos \theta$ $d \theta$. The projected area is therefore integrated and divided by the integral of the probability density. Because of symmetries, we can restrict the computation of the average $\bar{S}_{p}$ to $1 / 4$ of the total solid angle (sphere) covered by the variables $(\theta, \phi)$. Eq. 1 can be rewritten as Eq. 2 because of symmetries, mainly because a shadow 'from below' has the same area as a shadow 'from above' for any given shape. 


$$
\begin{aligned}
& \bar{S}_{p}=\frac{\int_{-\pi-\pi / 2}^{\pi} \int_{p}^{\pi / 2} S_{p}(\theta, \phi) 2 \pi \cos \theta d \theta d \phi}{\int_{-\pi}^{\pi} \int_{-\pi / 2}^{\pi / 2} 2 \pi \cos \theta d \theta d \phi}=\frac{1}{4 \pi} \int_{-\pi}^{\pi} \int_{-\pi / 2}^{\pi / 2} S_{p}(\theta, \phi) \cos \theta d \theta d \phi \\
& \bar{S}_{p}=\frac{1}{\pi} \int_{0}^{\pi} \int_{0}^{\pi / 2} S_{p}(\theta, \phi) \cos \theta d \theta d \phi
\end{aligned}
$$

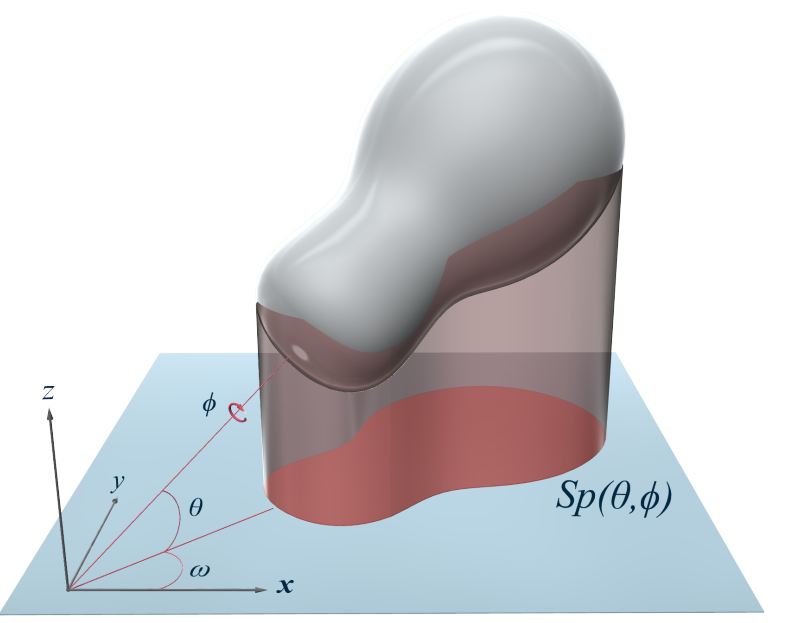

Figure 1: Illustration of a shape projection (in red below the white/gray shape) as performed by MoShade. The angles $\theta$ and rotation axis $\phi$ describe the shape's orientation and are sampled during the projection calculations. $\omega$ is the angle in the $(x, y)$ plane. The shape input is given as a mesh and the projected area is calculated as the sum of the projections of each of the mesh's triangles (see text).

If one wishes to use numerical integration instead of trying to integrate analytically, one may use Eq. 3 as simple quadrature of Eq. 2, with a uniform repartition of the $N$ samples on $\theta_{i}, \phi_{i} \in\left[0 \ldots \frac{\pi}{2}\right] \times[0 \ldots \pi]$. The $n$ samples parameterizing a MoShade calculation hence lead to $N=(2 n)^{2}$. 
$\bar{S}_{p}=\frac{1}{\pi} \int_{0}^{\pi} \int_{0}^{\pi / 2} S_{p}(\theta, \phi) \cos \theta d \theta d \phi \approx \frac{\pi}{2 N} \sum_{i} S_{p}\left(\theta_{i}, \phi_{i}\right) \cos \theta_{i}$

To evaluate $S_{p}\left(\theta_{i}, \phi_{i}\right)$, the surface of the shape must be decomposed into a mesh of simple geometrical elements (e.g. triangles or quads). Therefore MoShade uses mesh input files, where the shape is subdivided into small flat triangles (.stl files). The difficulty here is to compute the merged projected area for each triangle 'at once', i.e. by taking care of the shadowing of some triangles by others. MoShade therefore eliminates triangles that are not oriented adequately (see Supporting Information), and computes the intersection between the projection of each triangle and the current projected area for a given shape orientation $\left(\theta_{i}, \phi_{i}\right)$, and adds only the 'not already covered' area, if it exists.

However, directly using the quadrature in Eq. 3 yields numerical issues. Indeed, the result is inaccurate when integrating a constant cross-section coming from a spherical shape for instance. This bad behavior originates from the term in $\cos \theta$. One could expect an exact result even with only one sample point in this case, as for any reasonably good quadrature such as Gaussian integration. To reach this goal (i.e. an exact result), the integration is optimized using a change of variables (see Supporting Information for more details). The term $\cos \theta$ is cancelled in the integrand so that $\cos \theta d \theta$ is seen as constant. By changing $\theta=\sin ^{-1} \psi$, and by substituting the boundaries of the integral in Eq. 2 to their sinus, one obtains Eq. 4, which constitutes the optimized integration used by MoShade. Once again, the $n$ samples parameterizing a MoShade calculation lead to $N=(2 n)^{2}$.

$$
\bar{S}_{p}=\frac{1}{\pi} \int_{0}^{\pi} \int_{0}^{1} S_{p}\left(\sin ^{-1} \psi, \phi\right) d \psi d \phi \approx \frac{1}{N \pi} \sum_{i} S_{p}\left(\sin ^{-1} \psi, \phi_{i}\right)
$$


It should be noted that the change of variable works because the bounds of the integral are such that the application $\theta=\sin ^{-1} \psi$ is monotonous in the interval.

\section{MoShade input preparation and MoShade Automator.}

The model structure input geometries (.stl files) were prepared using Autodesk 123D Design 14.2.2, a free computer-aided design (CAD) software. Free software MoShade Automator (licensed GPLv2+), written in FreePascal (Object Pascal) using Lazarus, is a cross-platform (Linux and Windows 64-bit) graphical user interface for creating batch files of multiple structure inputs. It can also pilot MoShade by running, pausing or stopping the execution of MoShade between inputs of multiple batch files.

MoShade Automator can be downloaded at the following link:

https://github.com/JeanRNH/MoShadeAutomator/releases

\section{PEO structure pool.}

The PEO calculations, based on conventional computational chemistry, were performed on PEO DP $=14$ with 1 or 2 sodium cations. The structures were generated using both MM2 and MMFF94 force fields implemented in Chem3D Pro v.11.0. Structure optimizations and structure heating to $300 \mathrm{~K}$ or more were performed. Intermediately stopping the calculations and extracting the structures generated a diverse structure pool for the considered complexes. VMD ${ }^{39}$ 1.9.2 yielded conform .stl files for MoShade calculations ('QuickSurf' shape representation, resolution: 1.0, radius scale: 1.0, density isovalue: 0.3 , grid spacing: 1.0 ). 


\section{Results and Discussions}

\section{Polymers in IM-MS and experimental pow fitting parameters.}

Figure 2 plots the CCS as a function of the Degree of Polymerization (DP; converted from the mass-to-charge $\mathrm{m} / \mathrm{z}$ ratio) of PEO (5000 g/mol). The CCS evolution of highly charged polymer ions is generally increasing with increasing DP (e.g. $\left[\mathrm{PEO}+4 \mathrm{Na}^{+}\right]^{4+}$ to $\left.\left[\mathrm{PEO}+9 \mathrm{Na}^{+}\right]^{9+}\right)$. In different DP regions, the CCS decreases while the DP still increases (e.g. $\left[\mathrm{PEO}+4 \mathrm{Na}^{+}\right]^{4+} 93<\mathrm{DP}<100$ and $130<\mathrm{DP}<140$ ). During these disruptions in the generally increasing CCS evolutions, owing to an enhanced charge solvation inducing a decreased Coulomb repulsion with the elongating polymer chain, the three-dimensional structures of the complexes rearrange. After having undergone several structural rearrangements, the higher charge states merge with the most compact CCS evolution (i.e. the common trend line ${ }^{7,8,38}$ ), here represented by the $\left[\mathrm{PEO}+3 \mathrm{Na}^{+}\right]^{3+}$ complexes above DP 100.

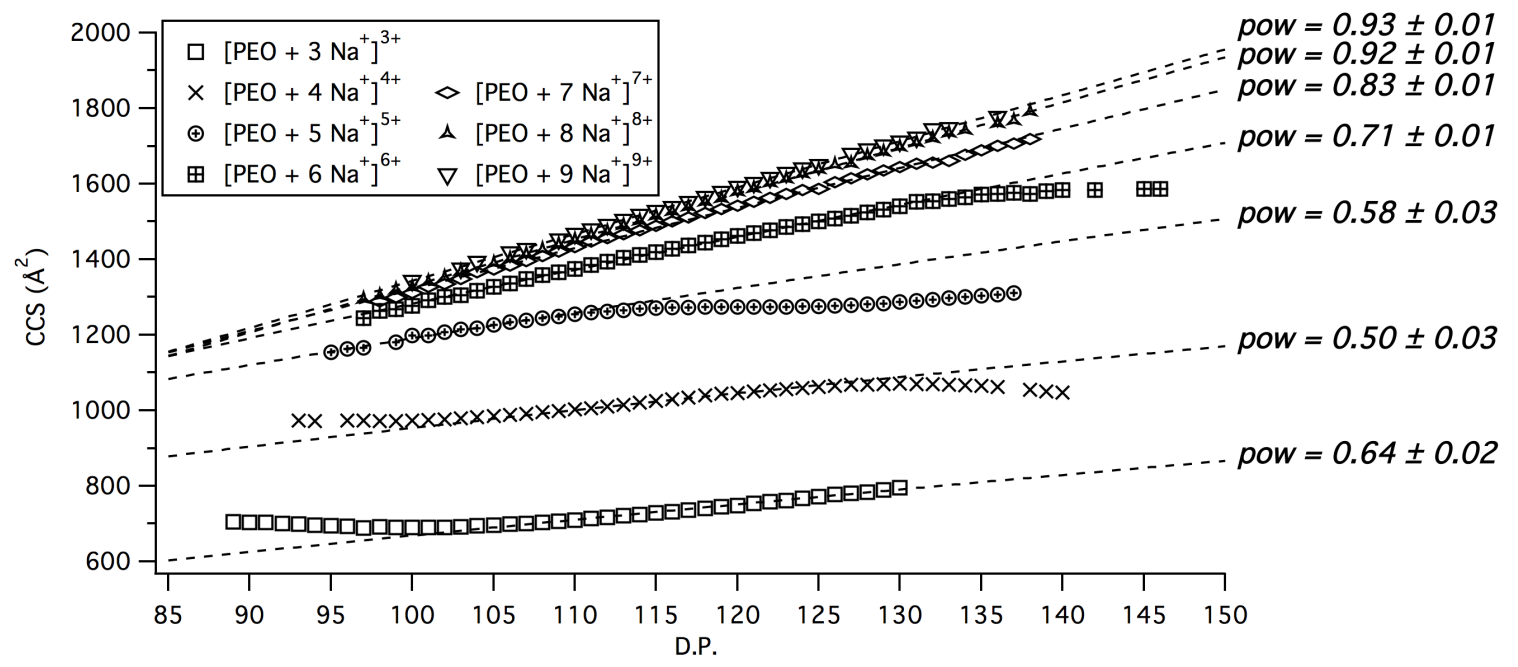

Figure 2: Collision Cross-Section (CCS) as a function of the Degree of Polymerization (DP) of poly(ethylene oxide) PEO. The charge states range from $3+$ to $9+$ using sodium 
cation adducts. The dotted lines represent the CCS evolution fits (Eq. 5) and the pow fit parameter of each fit is given.

The CCS evolutions before and after the structural rearrangements can be fitted using Eq. 5. In this study, we focused on the pow parameter. As we showed, it contains the shape information of the complexes. Interpretations on the parameter $A$ will be the focus of a specifically dedicated paper. When fitting the CCS evolutions ${ }^{38}$, the pow fit parameters of the polymer-cation complexes are found to roughly range from around 0.55 to 0.95 .

$\Omega=A \cdot D P^{\text {pow }}$

where $\Omega$ represents the CCS, $D P$ represents the Degree of Polymerization and $A$ and pow are the fitting parameters (as developed in another paper from our group ${ }^{38}$ ).

\section{Geometric structures and theoretical pow fitting parameters.}

The aim of the CCS calculations using MoShade is to investigate CCS evolutions of simple geometric shapes whose dimensions are perfectly tuned and controlled. From these shapes, controlled shape elongations and deformations are undertaken to study their effects on the CCS (i.e. CCS evolutions). In order to generate such model shapes, shapes were designed using a CAD software with no theoretical chemistry a prioris intervening. The known shape dimensions allow calculating the shape volume, which relates to the experimental polymer DP values and which enables our experiment-theory comparison. Additionally, MoShade calculates projections of the shapes without modeling any drift gas particles. The calculations take into account neither the mass nor the ion-drift gas particle interactions. The obtained CCS values, however, are related to an underlying apparent volume or the envelope of the ion-drift gas interactions as sensed by IM-MS. 
Table 1 summarizes the different shapes which were analyzed: spheres, cylinders and combinations of spheres and cylinders (Ball-Cylinder). The shape deformations, such as diameter increases, cylinder elongations or a uniform (isotropic) scaling of the entire shape, describe each considered case.

Table 1: Shape descriptions and shape deformations used to calculate CCS values and trends using MoShade. The shape variations were sampled as described in 'Dimensions'. The number of shapes sampled for each shape evolution is also provided (i). The pow parameters were extracted from fitting (Eq. 5) the plots of the CCS as a function of the shape volume. The volume was calculated using the geometry dimensions, taking into account the volume overlaps between two overlapping shapes (e.g. for Ball-Cylinder shapes) and was controlled using MoShade volume calculations. Schemes of the sampled shape evolutions are given and the CCS plots can be found in the Supporting Information (Figure S2 and Figure S3). Additional shapes are mathematically treated in the text.

\begin{tabular}{|c|c|c|c|c|c|}
\hline $\begin{array}{l}\text { Shape } \\
\text { description }\end{array}$ & $\begin{array}{l}\text { Shape variation } \\
\text { \& number of } \\
\text { shapes (i) }\end{array}$ & $\begin{array}{l}\text { Dimensions } \\
\text { (min. }- \text { max. }^{(\mathrm{a})}{ }^{(\mathrm{b})}\end{array}$ & pow $^{(\mathrm{c})}$ & Scheme $^{(\mathrm{d})}$ & Figure \\
\hline Sphere & $\begin{array}{l}\text { Diameter } \\
(\mathrm{i}=14)\end{array}$ & $5-250$ & $\begin{array}{l}0.66688 \\
\pm 8.26 \times 10^{-9}\end{array}$ & & S2.a. \\
\hline Cylinder & $\begin{array}{l}C L \\
(i=20)\end{array}$ & $\begin{array}{l}\text { CD: } 20 \\
\text { CL: } 5-300\end{array}$ & $\begin{array}{l}0.91308 \\
\pm 0.0243 \\
\text { or Linear fit }\end{array}$ & & S2.b. \\
\hline Cylinder & $\begin{array}{l}C D \\
(i=20)\end{array}$ & $\begin{array}{l}\text { CD: } 5-1000 \\
\text { CL: } 200\end{array}$ & $\begin{array}{l}0.79205 \\
\pm 0.0194\end{array}$ & & S2.c. \\
\hline Cylinder & $\begin{array}{l}C D \\
(i=10)\end{array}$ & $\begin{array}{l}\text { CD: } 5-125 \\
\text { CL: } 200\end{array}$ & $\begin{array}{l}0.57132 \\
\pm 0.0166\end{array}$ & I & S2.d. \\
\hline Ball-Cylinder & $\begin{array}{l}\mathrm{BD} \\
(\mathrm{i}=24)\end{array}$ & $\begin{array}{l}\text { BD: } 3.8-45 \\
\text { CD: } 3 \\
\text { CL: } 5\end{array}$ & $\begin{array}{l}0.65589 \\
\pm 0.00393\end{array}$ & & S3.a. \\
\hline Ball-Cylinder & $\begin{array}{l}\mathrm{BD} \\
(\mathrm{i}=15)\end{array}$ & $\begin{array}{l}\text { BD: } 3.8-9 \\
\text { CD: } 3 \\
\text { CL: } 5\end{array}$ & $\begin{array}{l}0.60632 \\
\pm 0.00267\end{array}$ & $\rightarrow$ & S3.b. \\
\hline
\end{tabular}




\begin{tabular}{|c|c|c|c|c|c|}
\hline Ball-Cylinder & $\begin{array}{l}\text { Uniform Scaling } \\
(i=17)\end{array}$ & $\begin{array}{l}\text { BD: } 1.6-60.9 \\
\text { CD: } 0.6-22.8 \\
\text { CL: } 0.7-26.6\end{array}$ & $\begin{array}{l}0.66694 \\
\pm 0.000362\end{array}$ & C & S3.c. \\
\hline Ball-Cylinder & $\begin{array}{l}\text { Uniform Scaling } \\
(\mathrm{i}=18)\end{array}$ & $\begin{array}{l}\text { BD: } 1.6-65.8 \\
\text { CD: } 0.6-24.7 \\
\text { CL: } 1.4-57.5\end{array}$ & $\begin{array}{l}0.66802 \\
\pm 0.00163\end{array}$ & & S3.c. \\
\hline Ball-Cylinder & $\begin{array}{l}\text { Uniform Scaling } \\
(\mathrm{i}=18)\end{array}$ & $\begin{array}{l}\text { BD: } 1.6-65.8 \\
\text { CD: } 0.6-24.7 \\
\text { CL: } 2.1-86.3\end{array}$ & $\begin{array}{l}0.66796 \\
\pm 0.00157\end{array}$ & & S3.c. \\
\hline Ball-Cylinder & $\begin{array}{l}\mathrm{CL} \\
(\mathrm{i}=21)\end{array}$ & $\begin{array}{l}\text { BD: } 20 \\
\text { CD: } 3 \\
\text { CL: } 3.5-125\end{array}$ & Linear fit & 8 & S3.d. \\
\hline
\end{tabular}

\footnotetext{
(a) $\mathrm{BD}$ represents the ball diameter, $\mathrm{CD}$ is the cylinder diameter and $\mathrm{CL}$ represents the cylinder length

(b) The dimensions are provided in arbitrary units

(c) The pow parameter is given with its $95 \%$ confidence interval (pow $\pm 95 \%$ confidence interval)

(d) The schemes are provided from the starting shape (on the left) to the final shape (on the right)
}

\section{Simple shapes: Spheres and cylinders.}

First, we consider the case of a perfect sphere whose diameter increases. As expected in literature $^{37,38,40}$, the CCS evolution of spheres results in pow $=2 / 3$ (or $\approx 0.66$, Table 1 , Figure S2.a). Mathematically, based on a simplified equation for a solid of revolution where only one degree of freedom $\theta$ remains (Eq. 6; see the development of Eq. S1), the projected area is constant and is given by Eq. 7. This then yields the average crosssection given by Eq. 8 (based on Eq. S1).

$$
\begin{aligned}
& \bar{S}_{p}=\frac{\int_{-\pi / 2}^{\pi / 2} S_{p}(\theta) 2 \pi \cos \theta d \theta}{\int_{-\pi / 2}^{\pi / 2} 2 \pi \cos \theta d \theta}=\frac{1}{2} \int_{-\pi / 2}^{\pi / 2} S_{p}(\theta) \cos \theta d \theta=\int_{0}^{\pi / 2} S_{p}(\theta) \cos \theta d \theta \\
& S_{p}=\pi \frac{D^{2}}{4}
\end{aligned}
$$

where $S_{p}$ is the projected area of a sphere for a given orientation and $D$ its diameter. 
$\bar{S}_{p}=\int_{0}^{\pi / 2} \pi \frac{D^{2}}{4} \cdot \cos \theta d \theta=\pi \frac{D^{2}}{4}$

where $\bar{S}_{p}$ is the average projected area (or average cross-section).

In addition, the volume of the sphere is given by $V=\pi \frac{D^{3}}{6}$. What is of interest here is the behaviour of the mean projected area with respect to the mass $m$. In our case, the volume is proportional to the mass $V \propto m$, so that $D \propto m^{1 / 3}$ and therefore $\bar{S}_{p} \propto m^{2 / 3}$ (cf. Eq. 8).

By comparing the theoretical and experimental pow values, the growing spheres describe the envelope of the ion-drift gas particle interactions of the common trend line (Figure 2 $\left.\left[\mathrm{PEO}+3 \mathrm{Na}^{+}\right]^{3+}\right)$

When considering a cylinder with spherical ends, the projected area again depends only on the angle $\theta$, given that it is also a solid of revolution (Eq. 6 or Eq. S1). For the spherical parts, the description is identical to the case of a sphere (see above) because the two half-spheres are present at the extremities. The cylindrical part does not shadow the spherical ends differently as if they were a simple sphere. For the cylindrical part, a term in $\cos \theta$ needs to be added in order to take into account the slope with respect to the projection plane (Eq. 9).

$S_{p}(\theta)=\pi \frac{D^{2}}{4}+D L \cos \theta$

where $S_{p}(\theta)$ is the projected area dependent on the angle $\theta, D$ and $L$ are the cylinder diameter and length, respectively.

Given the volume $V=\pi \frac{D^{3}}{6}+\pi \frac{D^{2}}{4} L$ of the shape, the average projected area (average cross-section) yields Eq. 10 (based on Eq. 6 or Eq. S1). 
$\bar{S}_{p}=\int_{0}^{\pi / 2}\left(\pi \frac{D^{2}}{4}+D L \cos \theta\right) \cdot \cos \theta d \theta=\pi \frac{D^{2}}{4}+D L \int_{0}^{\pi / 2} \frac{1+\cos 2 \theta}{2} d \theta=\frac{\pi}{4}\left(D^{2}+D L\right)$

In this case, as $V \propto m$ still holds, $D \propto L \propto m^{1 / 3}$ and therefore $\bar{S}_{p} \propto m^{2 / 3}$ if one considers that the increase in size is isotropic. No change with respect to the simpler spherical case is to be noted. This is the case for every convex shape.

However, if the cylinder with spherical ends increases in length without increasing in diameter, meaning that the aspect ratio changes, one finds a linear relation between the average cross-section and the mass: $D=$ constant and $L \propto m$, therefore $\bar{S}_{p} \propto m$. This also holds for cylinders with plain level ends whose length increases (Table 1, Figure S2.b). The elongating cylinder either yields pow $=0.91$ or can be fitted with a linear function. Such structures then represent the highly-charged ions $\left(\left[\mathrm{PEO}+8 \mathrm{Na}^{+}\right]^{8+}\right.$ and $[\mathrm{PEO}+$ $\left.\left.9 \mathrm{Na}^{+}\right]^{9+}\right)$.

For cylinders with spherical ends, if the diameter increases for a constant length, the behaviour is more complex: $L=$ constant and $D \propto m^{1 / 2}$, therefore yielding $\bar{S}_{p} \propto m^{1 / 2}$ if the diameter $D$ is small with respect to $L$. Such shapes are represented by $\left[\mathrm{PEO}+4 \mathrm{Na}^{+}\right]^{4+}$ ions (Figure 2). However, if $L$ is small with respect to $D$, then $D \propto m^{1 / 3}$ and therefore $\bar{S}_{p} \propto m^{2 / 3}$. This case resembles a distorted sphere.

For cylinders with plain level ends whose diameter increases for a constant length, an intermediate pow value of around 0.80 can be found if $L$ is small with respect to $D$ (Table 1, Figure S2.c). This would correspond to the CCS trend of $\left[\mathrm{PEO}+7 \mathrm{Na}^{+}\right]^{7+}$ sampled in Figure 2. Nevertheless, the fit yielding 0.80 for cylinder diameter variations is highly influenced by CCS values at large volumes ( $L$ is small with respect to $D$ ). If the diameter $D$ is small with respect to $L$, pow parameters which are smaller than 0.66 can be found 
(pow $=0.57$; Table 1, Figure S2.d). Such pow values would then correspond to $[\mathrm{PEO}+4$ $\left.\mathrm{Na}^{+}\right]^{4+}$ and $\left[\mathrm{PEO}+5 \mathrm{Na}^{+}\right]^{5+}$ complexes.

Finally, we can consider the effect of the thickness of a hollow sphere. If the increase in size is isotropic (increase in diameter and in thickness), it is the same procedure as for a simple sphere: only the volume $V$ changes. However, if considering that the thickness of the sphere stays constant (e.g. fullerenes ${ }^{41,42}$ ), the volume becomes proportional to its surface and to the thickness $e$ (considered very small). The average projected area (crosssection) of a simple sphere still holds $\bar{S}_{p}=\pi \frac{D^{2}}{4}$ (Eq. 8), with $V=\pi D^{2} e$ so that $D \propto m^{1 / 2}$ and therefore $\bar{S}_{p} \propto m$. This is the same behaviour as a cylinder of constant diameter that increases in length. It is therefore impossible to discriminate both cases by just comparing the exponent (of the 'mass' variable) of the CCS evolution fit as a function of the mass.

\section{Composite shapes: Ball-cylinders.}

Regarding composite shapes (sphere with cylinder), several size parameters can be varied. We considered the relative ball or cylinder diameters, as well as only the cylinder length and a uniform scaling of the entire shape.

For increasing ball diameters in ball-cylinder shapes, a pow parameter of 0.655 is found (Table 1, Figure S3.a). This result was expected as the cylinder becomes increasingly negligible with the largely growing sphere at high volumes, thus evolving like a sphere (0.66). However, the power fit is again highly influenced by the larger CCS values at large volume values. When fitting at small ball diameters, pow values of 0.60 can be reached (Table 1, Figure S3.b). In this case, the cylinder and the sphere have almost identical diameters and the shape resembles a cylinder. Indeed, values smaller than 0.66 were found for cylinder diameter variations when $D$ was small with respect to $L$ (small 
CCS values; Table 1, Figure S2.d). This ball-cylinder value can thus be explained solely on the basis of its spherical and cylindrical components and can be predicted. When uniformly scaling a ball-cylinder shape (isotropic growth), the pow parameters reach 0.67. Identical pow values were found for all the ball-cylinder scaling series with different initial cylinder lengths, even for cylinder lengths surpassing the ball diameter (Table 1, Figure S3.c). Given the experimental errors on the experimental fit parameters (Figure 2), this result indicates that IM-MS would be unable to differentiate a sphere with increasing diameter (Figure S2.a), a ball-cylinder shape with a large and increasing sphere diameter (Figure S3.a) or a ball-cylinder shape with an uniform and isotropic volume increase (Figure S3.c). Similar to the conclusions drawn on convex shapes, IMMS would then only be sensitive (i.e. different pow values) to anisotropic shape growths. Finally, we analyzed the effect of a cylinder length increase of a ball-cylinder shape on the CCS evolution (Table 1, Figure S3.d.). Not surprisingly, this resulted in a linear CCS evolution as a function of the volume, similar to an elongating cylinder. The coupled sphere does only account for an offset in CCS or volume, given that its dimensions are kept unchanged. Such shapes could be related to high charge states where several monomers bearing cation charges are subjected to strong Coulomb repulsions (cylinder segment of the ball-cylinder shape) and other monomers form a cation-solvating globule with little Coulomb repulsion (ball segment of the ball-cylinder shape).

Table 3 summarizes the results of the MoShade-obtained pow ranges for the evolutions of simple and composite shape deformations. 
Table 3: Summary of the shape evolutions correlated to their pow range. See text for more detailed pow values.

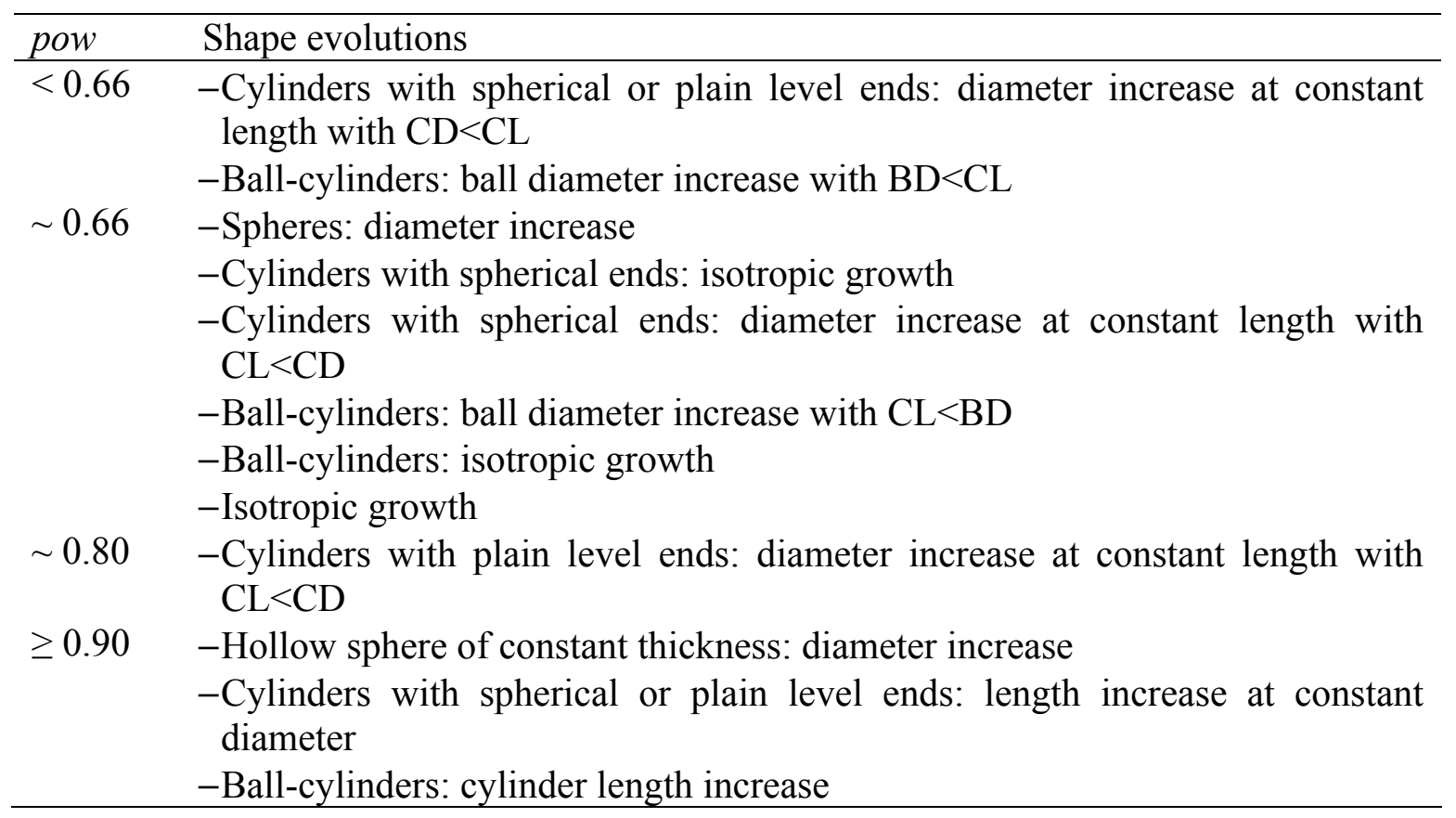

\section{Perfectly convex shapes: relation between surface area and CCS.}

\section{Theory.}

Owing to the well-defined and known geometries, different relations between the CCS and geometry parameters can be tested. One recurring relation which stands out is the relation between the (geometric) surface area of the shape and its CCS. Indeed, the geometric surface area equals $4 \times \mathrm{CCS}$ (Eq. 11). This can be mathematically demonstrated for perfectly convex shapes (spheres, cylinders; see Eq. S6 to Eq. S10 in the Supporting Information) and acts as a descriptor of the structure concavity.

$\bar{S}_{p}=\frac{S}{4} \quad$ or $S=4 \cdot C C S$

where $S$ is the area of the shape and $\bar{S}_{p}$ or CCS is the average cross-section. 
Even if this relation can only be mathematically demonstrated for perfectly convex structures but not for concave structures, it seems nevertheless also valid for the aboveanalyzed composite ball-cylinder shapes (Table 1). These shapes begin to exhibit concavities, but still yield surface-to-CCS ratios $S / C C S \leq 4.06$.

\section{Application to PEO.}

The CCS evolutions of the spheres, cylinders and ball-cylinder shapes, which are all in adequacy with the $S=4$.CCS relation, describe the 'natural' CCS evolution when following one given charge state as a function of the DP. This relation could then be used to facilitate the structure screening in conventional computational chemistry methods.

In order to check its applicability, we generated a pool of $\left[\mathrm{PEO} D \mathrm{DP}=14+1 \mathrm{Na}^{+}\right]^{1+}$ and $\left[\mathrm{PEO}_{\mathrm{DP}=14}+2 \mathrm{Na}^{+}\right]^{2+}$ structures using MM2 and MMFF94 force fields. The structures were heated to different temperatures in order to effectively sample the potential energy surface and were extracted with and without structure optimizations (convergence). The $\left[\mathrm{PEO}_{\mathrm{DP}=14}+1 \mathrm{Na}^{+}\right]^{1+}$ complexes were used to investigate different representations in $\operatorname{VMD}^{39}$ (VdW radii, Surf, QuickSurf,...). Owing to the lack of Coulomb repulsion ${ }^{37}$, the shapes of the $1+$ complexes are globular and the chosen three-dimensional representation (volume envelope) should yield $S / C C S$ ratios close to 4 . The most appropriate representation is described in the Materials and Methods section.

We sampled two globular and one, considered as aberrant manually-distorted elongated $\left[\mathrm{PEO}_{\mathrm{DP}=14}+1 \mathrm{Na}^{+}\right]^{1+}$ shapes. The globular shapes yielded $S / C C S=4.07$ whereas the elongated shape yielded $S / C C S=4.13$ (Table S1). The globular shapes leading to $S / C C S$ values closest to 4 can thus be correlated to energy-optimized structure geometries. 
Out of the 18 sampled structures for $\left[\mathrm{PEO}_{\mathrm{DP}=14}+2 \mathrm{Na}^{+}\right]^{2+}$ (Table S1), the 3 shapes represented in Figure 3 exhibited the lowest $S / C C S$ ratio. Shapes 1 and 4 led to $S / C C S=$ 4.09 and shape 10 led to $S / C C S=4.08$. They incidentally correspond to optimized or nearly-optimized structures using the MM2 and MMFF94 force fields.

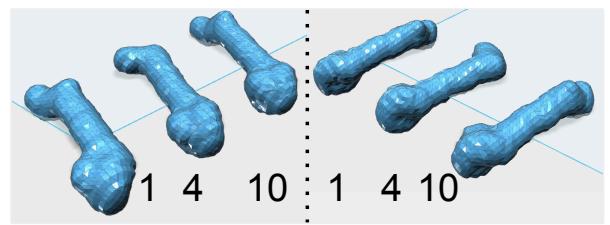

Figure 3: Shapes of $\left[\mathrm{PEO}_{\mathrm{DP}=14}+2 \mathrm{Na}^{+}\right]^{2+}$ (represented in 2 different viewing angles) leading to the lowest $S / C C S$ ratios out of all the 18 sampled shapes (see Table S1). Shape 1 and 4 yield $S / C C S=4.09$ and shape 10 yields $S / C C S=4.08$. Their underlying structures correspond to optimized or nearly-optimized structures using the MM2 and MMFF94 force fields.

Interestingly, even if the shapes extracted from conventional computational chemistry structures of PEO exhibit numerous concavities, the $S / C C S$ ratios closest to 4 are found for their optimized (or nearly-optimized) structures. Therefore, it seems that the S/CCS ratio calculation could be a new tool for rapid structure screening in conventional computational chemistry methods, as long as the shapes are not too concave. The limitations of structure filtering using the $S / C C S$ ratio due to concavities nevertheless still need to be explored in more detail.

It should still be noted that the $S / C C S$ relation is in fact independent of the CCS evolution. Indeed, one can calculate a $S / C C S$ ratio for any structure, outgrowing the starting point of polymer ions and the need to sample CCS evolutions instead of single- 
point absolute CCS values. In this case, experimental CCS measurements must then be accurate $e^{36,37}$ to calculate their surface area. The inspected surface area should then be accurately represented by the modeled 3D structures (e.g. by their electron density isosurfaces $\left.{ }^{33}\right)$ in order for them to pass the structure filter. Structure pools could then be 'randomly' generated using computational chemistry methods ${ }^{8,9,43-45}$ without the need for precise knowledge of experimental/simulation (ion or bath) temperatures. When finding suitable structures (using Eq. 11, S=4.CCS), the effective experimental ion temperature could then be retraced.

\section{Non-charge state-based CCS evolutions: Beads on a string.}

Besides following the CCS evolutions of fixed charge states as a function of the DP, CCS evolutions when varying the charge states can be analyzed. Literature predicts through theoretical chemistry that the highest charge states have a 'beads on a string' conformation $^{8,9}$. The beads on a string conformation would be made out of monomer beads each solvating a cation, and of monomer strings spacing the beads to reduce Coulomb repulsion. In our case, we created a CCS evolution, which is not based on a single fixed charge state, by experimentally considering only the first detected ion of each charge state, i.e., the ions with the smallest $m / z$ for a each $z$. These polymer ions exhibit the highest possible charge density. This thereby yields a structure evolution of beads on a string where every new complex has an additional 'bead-string' unit (or segment) for solvating the additional cation and Coulomb repulsion (see Figure 4.b).

In order to experimentally sample the first complexes of multiple charge states, PEO polymers were analyzed at small DP values, covering DP 8 to $\sim 70(750 \mathrm{~g} / \mathrm{mol}$ and 2000 
g/mol samples; Figure 4.a). Fitting this CCS evolution yields a pow value of around 0.93. It can also be fitted using a linear fit function which yields a slope of 13.9. Modeled beads on a string shapes, with different bead diameters and cylinder lengths or cylinder diameters, also yield linear CCS evolutions as a function of the volume. It should be noted that these structures do not follow the $S=4$.CCS relation described in Eq. 11 (S/CCS 4.10-4.40), given their increased concavity. Shape variations which both followed Eq. 11 and exhibited linear CCS evolutions could be associated to elongating cylinders (convex shapes). Nevertheless, we still considered the beads on a string structures as suggested by computational chemistry, given that our MoShade calculations can only yield 'coarse grain' information on the volume envelope of the ion-drift gas particle interactions and may falsely discard beads on a string atomic fine-structures.

When comparing bead-string units with identical volume but different cylinder dimensions (Figure 4.c 'BD 8 CD 3 CL 6' and 'BD 8 CD 2.12 CL 12'), the CCS values change and the slope changes. Thus, only specific (non-aberrant) bead-string dimensions can lead to these specific slope values. Nevertheless, the cylinder diameter can be changed with only a negligible effect on the slope (Figure 4.c, 'BD $20 \mathrm{CD} n$ ' with $\mathrm{n}=3$ or 6 and $\mathrm{CL}=3,6$ or 12). The cylinder length, which spaces each bead, and the bead diameter, leading to volume and CCS jumps, are the main parameters influencing the slopes. The beads on a string linear evolution can thus be compared to the MoShadeobtained 'coarse grain' cylinder elongations where the bead diameter defines the cylinder diameter and the string length defines the cylinder elongation. 

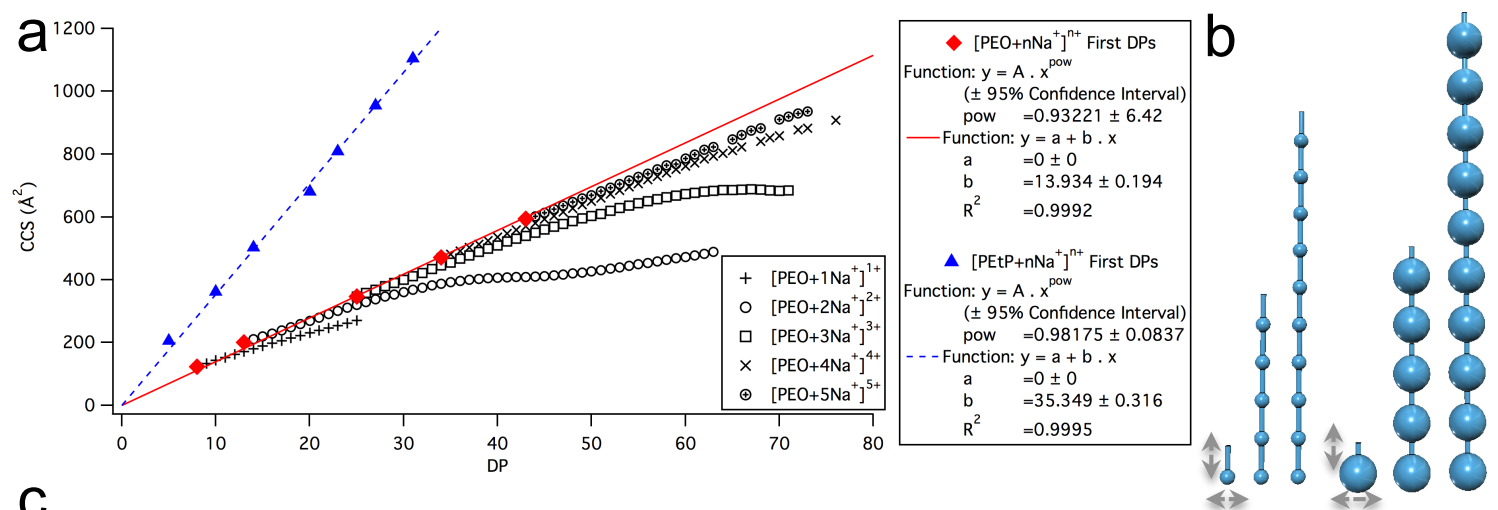

\section{C}

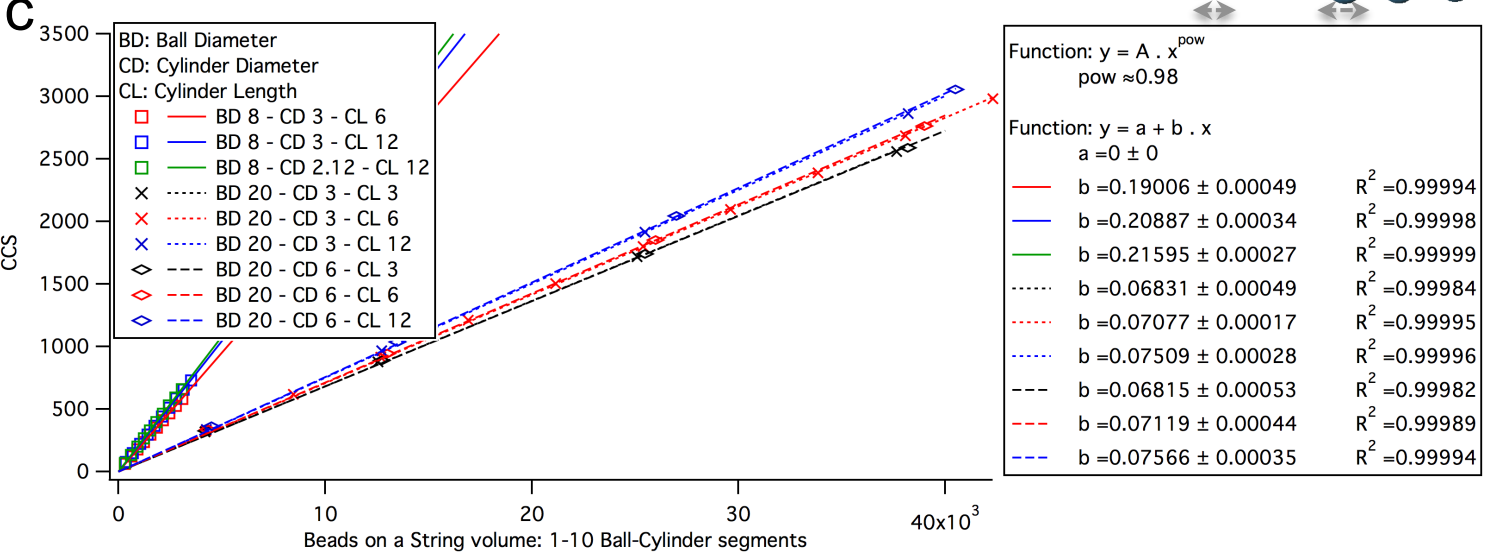

Figure 4: Comparison of the experimental CCS-DP plots considering only the lowest $\mathrm{m} / \mathrm{z}$ (or DP/z) adduct of each charge state of sodiated PEO and PEtP ions with the theoretical CCS-volume plots from CAD-constructed shapes computed using MoShade. 4.a Black markers depict the CCS evolution of PEO. The red markers represent the first PEO complex of each charge state, with the red plain line representing its linear fit function. This non-charge state-based CCS evolution equates a series of the highest-charged complexes. The blue markers depict the same series for PEtP polymers (see Figure S1), with its linear fit function depicted as blue dotted line. 4.b describes the modeled shape evolutions of such complexes: each considered complex is made up of an additional bead-string unit (or segment). 4.c Plot of the MoShade CCS (in arbitrary units) as a function of the volume of different beads on a string shapes. The linear fit coefficients 
and the coefficient of determination is given for the different fits. BD represents the ball diameter, $\mathrm{CD}$ represents the cylinder diameter and $\mathrm{CL}$ represents the cylinder length.

When extracting the same CCS evolution (first complex of each charge state) for a different polymer, poly(ethoxyphosphate) PEtP (Figure 4; PEtP scheme in Figure S1), a pow value of 0.98 can be attained. The slope of the linear fit yields a value of 35.3. The ratio of the PEtP and PEO slope values can then be calculated $(\mathrm{PEtP} / \mathrm{PEO}$ slope ratio $=$ 2.54; Table 2). Similar ratios can be calculated for all modeled beads on a string CCS evolution slopes (Table S2) and matching experimental and theoretical ratios can be found (Table 2). Two ratios of modeled beads on a string shapes resulted in close correlations with the experimental PEtP/PEO slope ratio. The only difference in the two modeled shape evolutions is the cylinder diameter of the beads on a string, as expected due to its lesser influence on the slope. We are thus able to provide relative bead-string dimensions of PEO compared to the PEtP bead-string units (or vice-versa). PEO should thus exhibit a bead diameter of 20 if PEtP exhibits a bead diameter of 8 (in arbitrary units). The cylinder length should be doubled when going from PEtP to PEO. 
Table 2: Slopes of the linear fits $(b)$ of modeled beads on a string shapes (see Figure 4.c) and of experimental PEtP and PEO evolutions of every first complex of each charge state (see Figure 4.a). The ratios of the modeled shapes are in close agreement with the experimental slope ratio PEtP/PEO. Table S2 lists all the modeled shapes with all the calculated slope ratios.

\begin{tabular}{|c|c|c|}
\hline $\begin{array}{l}\text { Bead-string unit } \\
\text { BD-CD-CL }\end{array}$ & $\begin{array}{l}b \\
\text { (slope) }\end{array}$ & $\begin{array}{l}b_{8-3-6} / b_{20-3-12} \\
b_{8-3-6} / b_{20-6-12}\end{array}$ \\
\hline $8-3-6$ & 0.190 & \\
\hline $20-3-12$ & 0.075 & 2.531 \\
\hline $20-6-12$ & 0.076 & 2.512 \\
\hline Polymer & $\begin{array}{l}b \\
\text { (slope) }\end{array}$ & $b_{P E t P} / b_{P E O}$ \\
\hline PEtP & 35.349 & \\
\hline PEO & 13.934 & 2.537 \\
\hline
\end{tabular}

Knowing these relations between the envelope dimensions of PEO and PEtP, theoretical chemistry modeling methods (MM, MD, DFT...) could find enhanced hypotheses or interaction potential descriptions for structure calculations. The considered number of PEO monomer units needs to fill up the given volume (or envelope) and if volume corrections need to be performed, proportional corrections need also to be applied to the PEtP volume envelope. The missing dimension, i.e. the cylinder diameter which only plays a minor role in the CCS evolution, will then be intrinsically defined through the width of the monomer units making up the string segment. We are hence able to give precise volume dimensions of the polymer ions without modeling any chemical interactions or atoms. 


\section{Conclusions \& Prospects}

The aim of this study was to link experimental CCS evolutions to potential geometric shape evolutions of the considered ions, without the need of time-consuming conventional computational chemistry methods and without needing accurate measured CCS values. We presented a new orthogonal method for relating experimental IM-MS measurements to theoretical shape interpretations, as opposed to using conventional computational chemistry. New software named MoShade allowed calculating CCS values of (mesh-represented) shapes through projection calculations, which yield the volume envelope of the ion-drift gas particle interactions. Our MoShade calculations were purely geometric shape-based analyses with no 3D structures from theoretical chemistry intervening in the process. No atoms or chemical interactions were modeled.

The study was experimentally based on synthetic homopolymer ions in order to obtain ions with constant interactions, and yielding CCS evolutions over large mass ranges. The CCS evolutions were fitted using power fit functions ${ }^{38}$, avoiding absolute CCS value interpretations. We showed here that the pow parameter in the CCS fit equation $C C S=A . D P^{p o w}$ contains the structural shape information accessible through IM-MS.

Basic shape geometries and shape variations were mathematically considered or computationally modeled. The modeled shape dimension variations yielded theoretical CCS trends, which were also fitted using power fit functions. We then established comparisons between the experimental and mathematically-obtained/modeled pow parameters. We were thus able to extract several shape evolutions which fitted with experimentally-obtained CCS evolutions of polymers (summary in Table 3). 
Generally, an isotropic growth of shapes should always exhibit a 2/3 pow value, as it can be mathematically demonstrated. IM-MS CCS evolutions (pow values) for such shape evolutions are thus indistinguishable.

For perfectly convex shapes (e.g. spheres, cylinders), we mathematically demonstrated that the geometric surface area is related to the CCS of the shape as $S=4$.CCS. Interestingly, energy-optimized MM structures (MM2 and MMFF94 force fields) of PEO ions exhibited the closest values to $S / C C S=4$ compared to non-optimized structures. This relation could then potentially act as structure filter for conventional computational chemistry methods, even for non-polymeric systems as it is applicable to absolute CCS values ${ }^{36,37}$ without the need for CCS evolutions. This relation should nevertheless still be studied in more detail and surface areas should be chosen wisely (e.g. electron density isosurfaces $^{33}$ ) in order to describe the volume envelope of the ion-drift gas interactions.

Finally, we investigated literature-advocated beads-on-a-string shapes ${ }^{8,9}$. We compared experimental data of two different polymers with CCS evolutions of modeled beads-on-astring shapes. MoShade enabled us to extract precise shape dimensions of the bead-string units for the two polymers (PEtP, PEO) without modeling any chemical interactions. Given that the number of monomer units making up the beads on a string shape volume is known (MS identification), theoretical chemistry interaction potentials could be refined using MoShade in conjunction with experimental CCS evolutions.

After having analyzed the CCS trends, we could now begin modeling the DP ranges of the structural rearrangements of polymer ions. Therefore, shape variations inducing tipping points in the modeled CCS trends should be sampled. A first attempt of one of the 
possible shape variations is shown in Table S3. Angle variations between composite ballcylinder shapes ( 3 balls and 2 cylinders) can lead to CCS decreases for constant volumes.

\section{Conflicts of interest}

There are no conflicts to declare.

\section{Acknowledgments}

The authors acknowledge financial support of the F.R.S.- FNRS (FRIA). Prof. C. Jérôme and Dr. P. Lecomte (CERM laboratory, University of Liège) are recognized for their support for the synthesis of PEtP.

\section{References.}

(1) Hines, K. M.; Ross, D. H.; Davidson, K. L.; Bush, M. F.; Xu, L. Anal. Chem. 2017, 89 (17), 9023-9030.

(2) Boschmans, J.; Lemière, F.; Sobott, F. J. Chromatogr. A 2017, 1490, 80-88.

(3) Massonnet, P.; Haler, J. R. N.; Upert, G.; Degueldre, M.; Morsa, D.; Smargiasso, N.; Mourier, G.; Gilles, N.; Quinton, L.; De Pauw, E. J. Am. Soc. Mass Spectrom. 2016, 27 (10), 1637-1646.

(4) López, A.; Tarragó, T.; Vilaseca, M.; Giralt, E. New J. Chem. 2013, 37 (5), 1283.

(5) Bleiholder, C.; Dupuis, N. F.; Wyttenbach, T.; Bowers, M. T. Nat. Chem. 2011, 3 (2), 172-177.

(6) Haler, J. R. N.; Far, J.; Aqil, A.; Claereboudt, J.; Tomczyk, N.; Giles, K.; Jérôme, C.; De Pauw, E. J. Am. Soc. Mass Spectrom. 2017, 28 (11), 2492-2499. 
(7) Haler, J. R. N.; Massonnet, P.; Chirot, F.; Kune, C.; Comby-Zerbino, C.; Jordens, J.; Honing, M.; Mengerink, Y.; Far, J.; Dugourd, P.; De Pauw, E. J. Am. Soc. Mass Spectrom. 2018, 29 (1), 114-120.

(8) Larriba, C.; Fernandez De La Mora, J. J. Phys. Chem. B 2012, 116 (1), 593-598.

(9) Trimpin, S.; Plasencia, M.; Isailovic, D.; Clemmer, D. E. Anal. Chem. 2007, 79 (21), 7965-7974.

(10) De Winter, J.; Lemaur, V.; Ballivian, R.; Chirot, F.; Coulembier, O.; Antoine, R.; Lemoine, J.; Cornil, J.; Dubois, P.; Dugourd, P.; Gerbaux, P. Chem. - A Eur. J. 2011, 17 (35), 9738-9745.

(11) Boschmans, J.; Jacobs, S.; Williams, J. P.; Palmer, M.; Richardson, K.; Giles, K.; Lapthorn, C.; Herrebout, W. A.; Lemière, F.; Sobott, F. Analyst 2016, 141 (13), 4044-4054.

(12) Counterman, A. E.; Clemmer, D. E. J. Phys. Chem. B 2002, 106 (46), 1204512051.

(13) Kinnear, B. S.; Kaleta, D. T.; Kohtani, M.; Hudgins, R. R.; Jarrold, M. F. J. Am. Chem. Soc. 2000, 122 (38), 9243-9256.

(14) Lapthorn, C.; Pullen, F. S.; Chowdhry, B. Z.; Wright, P.; Perkins, G. L.; Heredia, Y. Analyst 2015, 140 (20), 6814-6823.

(15) Shvartsburg, A. A.; Jarrold, M. F. Chem. Phys. Lett. 1996, 261 (1-2), 86-91.

(16) Mesleh, M. F.; Hunter, J. M.; Shvartsburg, A. A.; Schatz, G. C.; Jarrold, M. F. J. Phys. Chem. 1996, 100 (40), 16082-16086.

(17) Takaya, K.; Kaneko, T.; Tanuma, H.; Nishide, T.; Sugiyama, H.; Nakano, N.; Nagashima, H.; Seto, Y. Int. J. Ion Mobil. Spectrom. 2016, 19 (4), 227-232. 
(18) Larriba, C.; Hogan, C. J. J. Comput. Phys. 2013, 251, 344-36.

(19) Larriba, C.; Hogan, C. J. J. Phys. Chem. A 2013, 117 (19), 3887-3901.

(20) Marklund, E. G.; Degiacomi, M. T.; Robinson, C. V; Baldwin, A. J.; Benesch, J. L. P. Structure 2015, 23 (4), 791-799.

(21) Bleiholder, C.; Wyttenbach, T.; Bowers, M. T. Int. J. Mass Spectrom. 2011, 308 (1), 1-10.

(22) Morsa, D.; Gabelica, V.; De Pauw, E. Anal. Chem. 2011, 83 (14), 5775-5782.

(23) Merenbloom, S. I.; Flick, T. G.; Williams, E. R. J. Am. Soc. Mass Spectrom. 2012, $23(3), 553-562$.

(24) Liu, F. C.; Kirk, S. R.; Bleiholder, C. Analyst 2016, 141 (12), 3722-3730.

(25) Carpenter, J. E.; McNary, C. P.; Furin, A.; Sweeney, A. F.; Armentrout, P. B. J. Am. Soc. Mass Spectrom. 2017, 28 (9), 1876-1888.

(26) Hernandez, D. R.; DeBord, J. D.; Ridgeway, M. E.; Kaplan, D. A.; Park, M. A.; Fernandez-Lima, F. Analyst 2014, 139 (8), 1913-1921.

(27) Ujma, J.; Giles, K.; Morris, M.; Barran, P. E. Anal. Chem. 2016, 88 (19), 94699478.

(28) Giles, K.; Ujma, J.; Wildgoose, J.; Green, M. R.; Richardson, K.; Langridge, D.; Tomczyk, N. In 65th ASMS Conference on Mass Spectrometry and Allied Topics; 2017.

(29) Siems, W. F.; Viehland, L. A.; Hill, H. H. Analyst 2016, 141 (23), 6396-6407.

(30) Deng, L.; Webb, I. K.; Garimella, S. V. B.; Hamid, A. M.; Zheng, X.; Norheim, R. V.; Prost, S. A.; Anderson, G. A.; Sandoval, J. A.; Baker, E. S.; Ibrahim, Y. M.; Smith, R. D. Anal. Chem. 2017, 89 (8), 4628-4634. 
(31) Warnke, S.; Seo, J.; Boschmans, J.; Sobott, F.; Scrivens, J. H.; Bleiholder, C.; Bowers, M. T.; Gewinner, S.; Schöllkopf, W.; Pagel, K.; Von Helden, G. J. Am. Chem. Soc. 2015, 137 (12), 4236-4242.

(32) Kune, C.; Haler, J. R. N.; Far, J.; De Pauw, E. ChemPhysChem 2018, 19 (21), 2921-2930.

(33) Alexeev, Y.; Fedorov, D. G.; Shvartsburg, A. A. J. Phys. Chem. A 2014, 118 (34), $6763-6772$.

(34) Shrivastav, V.; Nahin, M.; Hogan, C. J.; Larriba-Andaluz, C. J. Am. Soc. Mass Spectrom. 2017, 28 (8), 1540-1551.

(35) Wu, T.; Derrick, J.; Nahin, M.; Chen, X.; Larriba-Andaluz, C. J. Chem. Phys. 2018, $148(7)$.

(36) Stow, S. M.; Causon, T. J.; Zheng, X.; Kurulugama, R. T.; Mairinger, T.; May, J. C.; Rennie, E. E.; Baker, E. S.; Smith, R. D.; McLean, J. A.; Hann, S.; Fjeldsted, J. C. Anal. Chem. 2017, 89 (17), 9048-9055.

(37) Haler, J. R. N.; Kune, C.; Massonnet, P.; Comby-Zerbino, C.; Jordens, J.; Honing, M.; Mengerink, Y.; Far, J.; De Pauw, E. Anal. Chem. 2017, 89 (22), 12076-12086.

(38) Haler, J. R. N.; Morsa, D.; Lecomte, P.; Jérôme, C.; Far, J.; De Pauw, E. Methods 2018, 144, 125-133.

(39) Humphrey, W.; Dalke, A.; Schulten, K. J. Mol. Graph. 1996, 14 (1), 33-38.

(40) Ruotolo, B. T.; Benesch, J. L. P.; Sandercock, A. M.; Hyung, S. J.; Robinson, C. V. Nat. Protoc. 2008, 3 (7), 1139-1152.

(41) Von Helden, G.; Hsu, M. T.; Gotts, N.; Bowers, M. T. J. Phys. Chem. 1993, 97 (31), 8182-8192. 
(42) Von Helden, G.; Hsu, M. T.; Kemper, P. R.; Bowers, M. T. J. Chem. Phys. 1991, $95(5), 3835-3837$.

(43) Schenk, E. R.; Nau, F.; Fernandez-Lima, F. Int. J. Ion Mobil. Spectrom. 2015, 18 (1-2), 23-29.

(44) Molano-Arevalo, J. C.; Jeanne Dit Fouque, K.; Pham, K.; Miksovska, J.; Ridgeway, M. E.; Park, M. A.; Fernandez-Lima, F. Anal. Chem. 2017, 89 (17), $8757-8765$.

(45) Schenk, E. R.; Almeida, R.; Miksovska, J.; Ridgeway, M. E.; Park, M. A.; Fernandez-Lima, F. J. Am. Soc. Mass Spectrom. 2015, 26 (4), 555-563. 